\title{
Sinais de risco para o suicídio em mulheres com história de violência
}

\section{doméstica*}

\author{
Cíntia Mesquita Correia ${ }^{1}$ \\ Normélia Maria Freire Diniz ${ }^{1}$ \\ Nadirlene Pereira Gomes ${ }^{1}$ \\ Isabela Carolyne Sena de Andrade ${ }^{1}$ \\ Luana Moura Campos ${ }^{1}$ \\ Jordana Brock Carneiro ${ }^{1}$
}

Objetivo: identificar sinais de risco para o suicídio em mulheres com história de violência doméstica. Método: estudo exploratório-descritivo, com abordagem qualitativa. Participaram dez mulheres com história de violência doméstica e tentativa de suicídio por envenenamento. $A$ pesquisa foi realizada no Núcleo de Estudo e Prevenção do Suicídio, vinculado a um Centro de Informação Toxicológica, em Salvador, Bahia, Brasil. Resultados: sinaliza para a relação entre a vivência de violência doméstica e o comprometimento para a saúde mental, representado por Comportamento depressivo e Comportamento suicida. Conclusão: o estudo revelou comportamentos que consistem em sinais de alerta para o risco de suicídio, ao tempo em que oferece subsídios para a promoção de estratégias de cuidado às mulheres com história de violência doméstica.

Descritores: Tentativa de Suicídio; Suicídio; Violência contra a Mulher; Violência Doméstica.

\footnotetext{
*Artigo extraído de dissertação de mestrado "Vivência de Violência Doméstica em Mulheres que Tentaram Suicídio" apresentada à Universidade Federal da Bahia, Salvador, BA, Brasil.

${ }^{1}$ Universidade Federal da Bahia, Salvador, BA, Brasil.
} 


\section{Signs of suicide risk in women with a history of domestic violence}

Objective: to analyze the meaning of the use and abuse of alcohol and / or other drugs among university students in accordance with Brazilian public policy on drugs. Methods: 49 university students from a Public Institution of Higher Education in the Southeast region of Brazil participated. Results: Bardin's Content Analysis elaborated the analytical category: "Dissonance between public policies and the meaning of drug use among university students". Drug use and abuse begin in adolescence; Stimulated by family and friends, and refers to the happiness and flight of problems. Conclusion: it is fundamental to know the meaning of drug use and abuse, from the perspective of the university for the elaboration and implementation of public policies.

Descriptors: Suicide, Attempted; Suicide; Violence against Women; Domestic Violence.

\section{Signos de riesgo para el suicidio en mujeres con antecedentes de violencia doméstica}

Objetivo: identificar signos de riesgo para el suicidio en mujeres con historia de violencia doméstica. Método: estudio exploratorio-descriptivo, con abordaje cualitativo Participaron diez mujeres con historia de violencia doméstica e intento de suicidio por envenenamiento. La investigación fue realizada en el Núcleo de Estudio y Prevención del Suicidio, vinculado a un Centro de Información Toxicológica, en Salvador, Bahia, Brasil. Resultados: señala para la relación entre la vivencia de violencia doméstica y el compromiso para la salud mental, representado por: Comportamiento depresivo y Comportamiento suicida. Conclusiones: el estudio reveló comportamientos que consisten en señales de alerta para el riesgo de suicidio, al tiempo que ofrece subsidios para la promoción de estrategias de cuidado a las mujeres con historia de violencia doméstica.

Descriptores: Intento de Suicidio; Suicidio; Violencia contra la Mujer; Violencia Doméstica.

Introdução

A violência doméstica contra a mulher é um fenômeno crescente em todo o mundo, configurando-se enquanto problema de saúde pública visto que acarreta adoecimento físico e mental, no qual se insere o suicídio.

Estimativas no país e no mundo apontam que muitas mulheres com história de violência apresentam ideação suicida. Pesquisa de base populacional, realizada na Suécia, com 205 mulheres, mostrou uma associação entre a violência doméstica e o comportamento suicida em $53 \%$ dos $\operatorname{casos}^{(1)}$. Na mesma direção, estudo norte americano sobre violência conjugal e ideação suicida, realizado com 166 gestantes na faixa etária de 16 a 28 anos, mostra uma relação para os pensamentos de morte e os abusos em $86 \%$ das participantes ${ }^{(2)}$. No Brasil, pesquisa sobre óbitos por suicídio realizada em dez municípios também revelou a violência doméstica como relacionada ao fenômeno(3). 
Quando não morrem por essa causa, as mulheres podem apresentar sequelas de natureza física e psicológica relacionadas às tentativas de suicídio. Isso porque o intento contra a própria vida pode provocar consequências bastante dolorosas e de tratamento prolongado, a exemplo das feridas por queimadura

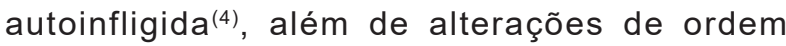
fisiológica, como sobrecarga hepática para os casos de intoxicação por medicamentos ${ }^{(5)}$. Dentre os aspectos psicossomáticos, destacam-se apatia, desesperança, fadiga, irritabilidade, insônia, inapetência, cefaleia e dores no corpo $^{(6-8)}$.

Vale salientar que tanto o adoecimento quanto a morte dessas mulheres interferem na produtividade do país, tendo em vista os anos potenciais de vida perdidos, os custos com tratamento, além do aumento na concessão de benefícios financeiros gerados à Seguridade Social. No México, estudo com 50 mulheres em situação de violência doméstica atendidas em um Centro Multidisciplinar de Atenção Integral à Violência desvelou o adoecimento em decorrência da violência e o desencadeamento do comportamento suicida em $58 \%$ das vítimas ${ }^{(9)}$. Essa realidade adverte para o elevado gasto público em internações e tratamentos direcionados ao reestabelecimento da saúde das mulheres, sinalizando para a necessidade de olhares profissionais mais atentos, sobretudo no sentido de prevenir tais situações ou mesmo identificá-las precocemente.

Ainda no que tange ao reconhecimento precoce da vivência de violência doméstica e/ou do evento suicida, dados do Ministério da Saúde alertam que $40 \%$ das pessoas que cometeram suicídio procuraram os serviços de saúde pelo menos 15 dias antes do desfecho fatal ${ }^{(10)}$. Além disso, sabe-se que, em decorrência das marcas físicas e psicológicas da vivência de violência, comumente essas mulheres são atendidas no âmbito secundário, nas emergências dos hospitais ou na atenção primária, em unidades básicas de saúde e centros de atenção psicossocial. Tendo em vista que esses espaços se constituem enquanto privilegiados para identificação precoce dos agravos, mostram-se essenciais o conhecimento e a sensibilidade profissional para a identificação de sinais de risco para o suicídio em mulheres com história de violência doméstica.

Nessa perspectiva é que nos questionamos: quais os sinais de risco para o suicídio em mulheres com história de violência doméstica? Adotamos como objetivo identificar os sinais de risco para o suicídio em mulheres com história de violência doméstica.

\section{Método}

Trata-se de um estudo exploratório-descritivo, com abordagem qualitativa. O estudo exploratório-descritivo adequa-se ao estudo, pois possibilita a elucidação e a descrição de um dado fenômeno(11), a exemplo do risco de suicídio em mulheres com história de violência doméstica. Esse fenômeno pode ser ainda melhor compreendido através da abordagem qualitativa, por permitir sua interpretação a partir das aspirações, atitudes, opiniões, percepções, crenças e valores extraídos de cada pessoa em sua convivência social(12).

Esta pesquisa foi realizada no Núcleo de Estudos e Prevenção do Suicídio (Neps), vinculado a um Centro de Informação Toxicológica (Ciave), localizado em Salvador, Bahia, Brasil. O Ciave é referência estadual na orientação aos casos de intoxicação exógena, incluindo os acidentes por animais peçonhentos e as tentativas de suicídio por envenenamento que são assistidos por serviços de saúde da rede pública e privada, bem como a população em geral.

Participaram da pesquisa dez mulheres adultas com história de violência doméstica e tentativa de suicídio por envenenamento, acompanhadas no Neps. Para a aproximação com as mulheres, realizou-se a ambientação do serviço por observações na sala de espera e participação em atividades de grupo realizadas pelo serviço de psicologia, no período de um mês, de segunda a sexta-feira, em turnos alternados entre o matutino e o vespertino.

Após ambientação, contou-se com o apoio da coordenação de psicologia do Neps para a identificação das mulheres favoráveis a participar do estudo, considerando-se os seguintes critérios de inclusão: idade igual e/ou maior a 18 anos; em acompanhamento psicológico e/ou psiquiátrico no Neps há, pelo menos, um ano; e em condições de estabilidade emocional e psíquica. Foram excluídas as mulheres que passaram por internação psiquiátrica nos últimos seis meses que antecederam a pesquisa. Todas as mulheres selecionadas foram convidadas a colaborar com o estudo e previamente esclarecidas quanto ao risco relacionado à participação na pesquisa, diante a possibilidade de sofrimento ao partilhar a sua história de violência e tentativa de suicídio. Tendo em vista tal situação, foi-lhes assegurado atendimento psicológico ou psiquiátrico imediato pelos profissionais que atuam no Neps.

As participantes foram ainda informadas quanto ao direito de desistir, em qualquer momento da pesquisa, sem prejuízos para o seu atendimento junto ao Neps. Além disso, comunicamos acerca dos benefícios da sua participação, que se refere à contribuição na produção 
do saber sobre as temáticas de violência doméstica e suicídio, bem como o direcionamento de ações para a melhoria da qualidade de vida das mulheres que as vivenciam.

Realizada a etapa de esclarecimentos, dez mulheres assinaram o Termo de Consentimento Livre e Esclarecido, conforme preconiza a Resolução 466/2012, do Conselho Nacional de Saúde, que regulamenta a ética em pesquisa em seres humanos. Esta pesquisa foi encaminhada para apreciação junto ao Comitê de Ética em Pesquisa do Hospital Ana Neri e aprovada sob o parecer $n^{\circ} 50 / 2010$.

Foram realizadas entrevistas semiestruturadas, utilizando-se como instrumento de coleta de dados um formulário que continha dados da caracterização sociodemográfica e uma pergunta aberta acerca da vivência de violência e das repercussões para a saúde mental. As falas foram gravadas e transcritas na íntegra, sendo o material arquivado, por até cinco anos, em pastas virtuais do Grupo de Estudos Violência, Saúde e Qualidade de Vida (Vid@). Para preservar a identidade das integrantes, foram atribuídos codinomes através da letra "E" e o numeral arábico correspondente à entrevistada.

Em seguida, os dados foram codificados com base na Análise Temática de Bardin. Esta visa obter, por meio de procedimentos sistemáticos e objetivos de descrição, o conteúdo das mensagens, indicadores (quantitativos ou não) que permitem a interferência de conhecimentos relativos às condições de produção/recepção dessas mensagens $^{(13)}$.

A operacionalização da análise temática descrita por Bardin seguiu três polos cronológicos, a saber: a pré-análise, que consiste na leitura exaustiva do material bruto; a exploração do material com consequente codificação dos dados e escolha das unidades de registro, que permitiu emergir as categorias de análise; e por fim o tratamento dos resultados por interpretação e inferência ${ }^{(13)}$. As categorias de análise que emergiram desse estudo foram Comportamento depressivo e Comportamento suicida.

\section{Resultados}

As mulheres que compuseram a amostra do estudo encontram-se na faixa etária entre 26 e 58 anos e, em sua maioria, são casadas e/ou convivem em união consensual. Apesar de quatro mulheres possuírem renda própria, por exercerem trabalhos remunerados fora de casa, apenas uma afirmou ter condições de assumir as responsabilidades financeiras sem a contribuição do companheiro. Todas as colaboradoras do estudo apresentaram de uma a quatro tentativas de suicídio por envenenamento através do uso de medicamentos e/ou raticidas, bem como narraram episódios de violência doméstica expressas nas formas física, sexual, patrimonial, moral ou psicológica.

Com relação aos sinais de alerta para o risco de suicídio, o estudo revela para a relação entre a vivência de violência e o comprometimento para a saúde mental, expresso através das categorias Comportamento depressivo e Comportamento suicida.

\section{Comportamento depressivo}

Sinais de comportamento depressivo, tais como labilidade emocional e baixa autoestima, foram evidenciados nas mulheres com história de violência doméstica e tentativa de suicídio. Esse quadro pode desencadear o sentimento de impotência diante da vida, comprometendo também o desenvolvimento das atividades laborais. Meu marido me humilhava em tempo integral. [...] dizia que eu não prestava para nada. [...] eu me sentia um lixo. Sempre me pegava chorando, me sentia inferior (E3); Ele nunca tocou em mim, mas ouvi palavras muito duras durante todos esses anos. Fui colocada no mais baixo nível. Me sentia fraca, pequena, incapaz. [...] não conseguia mais trabalhar. Não suportava mais nada. [...] já estava a ponto de enlouquecer (E7).

\section{Comportamento suicida}

Embora nem todas as mulheres com história de violência doméstica que manifestem sinais depressivos apresentem ideação suicida, o estudo sinaliza a possibilidade de evolução para condutas suicidas, como as tentativas de suicídio ou o suicídio propriamente dito. Estes podem ser evitados através do reconhecimento prévio de sinais característicos do comportamento suicida: rigidez do pensamento, impulsividade e ambivalência ${ }^{(14)}$.

\section{Rigidez do pensamento}

Diante do sofrimento psíquico decorrente da violência doméstica, algumas mulheres apresentam ideação constante de morte como a única solução para os problemas, incluindo-se recorrentes tentativas de suicídio. Meu marido me abusava sexualmente e eu ficava angustiada. [...] pensava que morrendo acabaria com tudo. [...] tentei suicídio três vezes. Vivia em prol disso (E3); Ele dizia que não me amava, que iria me trocar por duas mulheres de 15 anos. Em dois meses, tentei suicídio três vezes. [...] ia ao mercado e pensava em me atirar embaixo do ônibus. [...] 
passava por uma passarela e pensava de novo em suicídio, em me jogar lá de cima (E5).

\section{Impulsividade}

Enquanto algumas mulheres com histórias de tentativas de suicídio demonstram ideias e planos constantes de morte, outras agem também de forma impulsiva, principalmente diante situações conflitantes. Meu marido saiu para trabalhar e já tinha me dito um monte de coisa [...] até que eu era louca. Esperei ele sair e tomei vários comprimidos de Rivotril (E3); A primeira tentativa de suicídio foi depois de uma briga. [...] tomei dois frascos de chumbinho (raticida) (E10).

\section{Ambivalência}

Independentemente da rigidez de pensamento ou impulsividade, a urgência para sair de uma vida de violência é percebida nas falas de forma conflitante, por pensamentos ambivalentes entre os desejos de viver e os de pôr fim à dor psíquica. Assim, mesmo em face da exposição ao risco de morte, com a tentativa de suicídio, as mulheres não expressam claramente o desejo de morrer, mas o de livrar-se do sofrimento a qualquer custo. Depois da primeira tentativa, me arrependi e fiquei feliz por não ter morrido, por ter recebido a oportunidade de viver de novo [...] pensei que tudo poderia ser diferente, que conseguiria me livrar da dor (E7); Sentia uma dor muito grande, de ficar desesperada por ajuda. [...] tomei vários comprimidos para perder a consciência e me libertar da situação. Eu só queria dormir para esquecer a dor (E1).

\section{Discussão}

O estudo mostra a relação entre vivência de violência doméstica por mulheres e comportamento depressivo, comumente expresso por choro recorrente e baixa autoestima que compromete as atividades diárias, inclusive o desempenho profissional. Indisposição para trabalhar e quadros depressivos, caracterizados por tristeza, sentimentos de inferioridade e apatia, também foram evidenciados em mulheres inseridas em um cotidiano de violência no âmbito doméstico(15-16).

Uma vez imersas em sintomas depressivos, as mulheres podem desenvolver comportamento suicida, realidade vivenciada pelas participantes. Estudo realizado em Uganda, na África, relacionando violência e depressão, confirma a associação entre este agravo e a tentativa de suicídio(17). A interface do experienciar violência doméstica com o comportamento depressivo e suicida também foi apontada em pesquisa realizada nos
Estados Unidos da América(18). Nesses casos, percebese a ocorrência de elementos depressivos como forte preditor para o comportamento suicida.

Vale salientar que o estado depressivo desencadeia na mulher um sentimento de dor provocado pelas marcas da violência. Essa dor é traduzida por um sofrimento psíquico tão intenso que, em alguns casos, a morte é pensada como a melhor saída ${ }^{(19)}$. Assim, na tentativa de findar a dor, considerada insuportável, o suicídio apresenta-se como única alternativa. Daí a necessidade de compreender que a ideia de morte surge como um apelo desesperado por ajuda, sendo necessário refletir sobre o que está por trás desse ato ${ }^{(20)}$. Nesse contexto, a averiguação acerca da vivência de violência doméstica torna-se imprescindível.

Faz-se importante ainda referir sobre o pensamento contínuo ou impetuoso da ideação suicida perante as frustrações vivenciadas no âmbito doméstico. A primeira situação, rigidez do pensamento, caracteriza-se pela ideação constante de morte, podendo ser identificada precocemente, a partir da investigação da reação da mulher diante das situações difíceis ou extremas de vida, a exemplo dos conflitos conjugais. Nesses casos, a dificuldade para se pensar em outras possibilidades de saída do sofrimento, assinalada pela rigidez do pensamento, confere ao suicídio a única via para a resolução de problemas.

Na ocorrência de sinais depressivos, a tentativa de suicídio também pode ser desencadeada por atos de impulsividade ${ }^{(21)}$, ainda que as condutas suicidas indiquem, em sua maioria, um comportamento gradativo e progressivo iniciado por ideias e planos de morte. Assim, à medida que eventos negativos ocorrem, a impulsividade pode surgir, explosivamente, como uma resposta a experiências aversivas momentâneas, sem uma premeditação do ato(22). Essa especificidade, embora dificulte intervenções impeditivas de um desfecho de morte diante das tentativas de suicídio, pode corroborar para a prevenção de novas tentativas, haja vista serem esses eventos preditores do risco de suicídio(23).

Esteja relacionada à rigidez de pensamento ou à impulsividade, paradoxalmente à ideação suicida, percebe-se o desejo de viver. Nota-se essa ambivalência acerca da vida e da morte, por exemplo, na demonstração de sensação de alívio quando a morte não é concretizada. A associação GoodTerapy, que reúne profissionais especialistas em saúde mental de mais de 30 países, defende que a maioria das pessoas que pensa em suicídio não quer morrer, mas deseja livrar-se da dor experenciada(24).

A partir dessa expressão de ambiguidade entre o viver e morrer, bem como as demais características já 
apresentadas, os profissionais da saúde, em especial a enfermeira, podem atentar-se sobre o risco de suicídio em qualquer atendimento ou espaço de convívio com mulheres. Exemplo disso é partilhado em estudo realizado na Noruega com enfermeiras de emergências psiquiátricas, que alcançaram uma redução nas mortes por suicídio a partir da identificação de sinais de risco para o comportamento suicida ${ }^{(25)}$. Nesse sentido, urge o preparo profissional para o reconhecimento do indivíduo com potencial de risco, em especial quando já identificada história de violência doméstica, sobretudo buscando agir de forma preventiva e promovendo estratégias de cuidado em saúde mental.

\section{Conclusão}

O estudo revelou que os comportamentos depressivo e suicida consistem em sinais de alerta para o risco de suicídio em mulheres com história de violência doméstica. Sinalizou ainda que o quadro de depressão, expresso pelo choro recorrente, baixa autoestima e apatia, pode evoluir para a ideação suicida, ou ainda permeá-lo, de modo que tais sintomas merecem atenção por parte dos profissionais de saúde.

Aos sintomas depressivos somaram-se as características psicopatológicas conferidas ao comportamento suicida: rigidez do pensamento, impulsividade e sentimento ambivalente de morte. Estas, consideradas oriundas de um adoecimento mental progressivo, podem aparecer de forma sutil ou repentina, nesse último caso requerendo maior atenção para seu reconhecimento.

Não podemos deixar de mencionar que, independentemente das características apresentadas, a história de violência doméstica pode ser considerada importante preditor na investigação de risco para o suicídio. Desse modo, os profissionais da saúde, sobretudo os que assistem em todos os níveis de atenção, a exemplo dos profissionais da área de enfermagem, devem, em qualquer ambiente de aproximação e acolhimento a mulheres em situação de violência, averiguar a existência de sinais que favoreçam a ocorrência de tal agravo. Com isso, o estudo oferece subsídios para a promoção de estratégias visando à identificação precoce dos sinais de risco para o suicídio, o que pode evitar a consumação do ato suicida, bem como o cuidado à saúde mental das mulheres com história de violência doméstica.

É importante ressaltar que o suicídio não decorre de um ato fortuito, expressando-se, muitas vezes, como a única alternativa para a solução de um sofrimento intenso, insuportável e interminável. Sendo assim, a vivência de violência doméstica, bem como o comportamento depressivo, que se apresentam nas histórias das mulheres deste estudo, refletem a complexidade multidimensional de atentar contra a própria vida. Dessa forma, o estudo limita-se por não fazer associações entre a vivência de violência doméstica e o risco de suicídio, sendo necessária a investigação de outros fatores de risco.

\section{Referências}

1. Dufort M, Stenbacka M, Gumpert MC. Physical domestic violence exposure is highly associated with suicidal attempts in both women and men. Results from the national public health survey in Sweden. Eur J Public Health. [Internet]. 2015;25(3):413-8. [cited Jun 4 2017). Available from: https://www.ncbi.nlm.nih.gov/pmc/articles/PMC4440449/ pdf/cku198.pdf

2. Alhusen JL, Fronhman N, Purcell G. Intimate partner violence and suicidal ideation in pregnant women. Arch Womens Ment Health. [Internet]. 2015;18(4):573-8. [cited Jan 10 2017). Available from: https://www.ncbi.nlm.nih.gov/ pmc/articles/PMC4506210/pdf/nihms-670780.pdf

3. Cavalcante FG, Minayo MCS, Meneghel SN, Silva RM, Gutierrez DMD, Conte M, et al. Psychological and psychosocial autopsy on suicide among the elderly: a methodological approach. Ciênc Saúde Coletiva. 2012 Ago;17(8):2039-52. doi: http://dx.doi.org/10.1590/S141381232012000800015

4. Maciel KV, Castro EK, Lawrenz P. Os Motivos da Escolha do Fogo nas Tentativas de Suicídio Realizadas por Mulheres. Temas Psicol. [Internet]. 2014;22(1):195-206. [cited Jun 10 2017). Available from: http://pepsic.bvsalud. org/pdf/tp/v22n1/v22n1a15.pdf

5. Bravo V, Román M, Bettini M, Cerda P, Mieres JJ, Paris $E$, et al. Caracterización de la ingestión por sobredosis de paracetamol: Reporte de un centro de información toxicológica chileno. Rev Méd Chile. 2012 Mar;14(3):313-8. doi: http://dx.doi.org/10.4067/S0034-98872012000300005 6. Bittar D, Kohlsdorf M. Ansiedade e depressão em mulheres vítimas de violência doméstica. Psicol Argum. [Internet]. 2013;31(74):447-56. [cited 10 mar 2017]. doi: http://dx.doi.org/10.7213/psicol.argum.31.074.DS08

7. Silva DSD, Tavares NVS, Alexandre ARG, Freitas DA, Brêda MZ, Albuquerque MCS, et al. Depression and suicide risk among Nursing professionals: an integrative review. Rev Esc Enferm USP. 2015 Dec;49(6):1023-31. doi: http:// dx.doi.org/10.1590/S0080-623420150000600020

8. Tiwari A, Chan KL, Cheung DST, Fong DYT, Yan ECW, Tang DHM. The differential effects of intimate terrorism and situational couple violence on mental health outcomes among abused Chinese women: a mixed-method study. 
BMC Public Health. [Internet]. 2015;31(15):1-12. [cited Mar 10 2017]. Available from: https://www.ncbi.nlm.nih.gov/pmc/ articles/PMC4458009/pdf/12889_2015_Article_1649.pdf 9. Aguilar KKG, Chiapas JMR, Ibarra IPT. Suicide Risk in Female Victims of Domestic Violence in Mexico. Salud Pública Méx. [Internet]. 2013;55 (6):555-6. [cited Mar 10 2017]. Available from: https://www.scielosp.org/pdf/ spm/2013.v55n6/555-556/en

10. Ministério da Saúde (BR). Portaria $n^{\circ} 1.876$, de 14 de agosto de 2006. Diretrizes Nacionais para Prevenção do Suicídio. Diário Oficial [da] República Federativa do Brasil. 2006 ago. 14. Seção 65. p.1. [Acesso 10 jan 2017]. Disponível em: https://www.jusbrasil.com.br/diarios/687743/ pg-65-secao-1-diario-oficial-da-uniao-dou-de-15-08-2006 11. Gonçalves HA. Manual de Metodologia da Pesquisa Científica. 2 ed. São Paulo: Avercamp; 2014. 168p.

12. Minayo MCS. O desafio do conhecimento - pesquisa qualitativa em saúde. 14 ed. São Paulo: Hucitec; 2014. $468 p$.

13. Bardin L. Análise de conteúdo. São Paulo: Edições 70; 2011. 229p.

14. World Health Organization . Preventing suicide: a global imperative. [Internet]. Geneva: WHO Library Cataloguing-in-Publication Data; 2014 [cited 2017 Jan 10]. Available from: http://apps.who.int/iris/bitstream/han dle/10665/131056/9789241564779_eng.pdf;jsessionid= D4C6E077F6836F77E0BCBDDAB77799B8? sequence=1

15. Netto LA, Moura MAV, Queiroz ABA, Tyrrell MAR, Bravo MMP. Violência Contra a Mulher e suas Consequências. Acta Paul Enferm. [Internet]. 2014;27(5):458- 64. [Acesso 10 jan 2017]. Disponível em: http://www.scielo.br/pdf/ape/ v27n5/pt_1982-0194-ape-027-005-0458.pdf

16. Correia CM, Gomes NP, Couto TM, Rodrigues AD, Erdmann AL, Diniz NMF. Representations About Suicide Of Women With History Of Domestic Violence And Suicide Attempt. Texto Contexto Enferm. [Internet]. 2014; 23(1): 118-25. [cited 2017 May 10]. Available from: http://www. scielo.br/pdf/tce/v23n1/0104-0707-tce-23-01-00118.pdf 17. Kinyanda E, Hoskins S, Nakku J, Nawaz S, Patel V. Risk of major depressive disorder among older persons living in HIV-endemic central and southwestern Uganda. AIDS Care. [Internet]. 2016;28(12):1516-21. [cited 2017 May 10]. Available from: http://researchonline.Ishtm. ac.uk/2551492/1/Risk\%20of\%20major\%20depressive\%20 disorder\%20among\%20older\%20persons_GREEN\%20 AAM.pdf

18. Beydoun MA, Kaufman JS, Lo B, Zonderman AB. Intimate partner violence against adult women and its association with major depressive disorder, depressive symptoms and postpartum depression: systematic review and meta-analysis. Soc Sci Med. [Internet]. 2012;75(6):95975. [cited 2017 May 10]. Available from: https://www.ncbi. nlm.nih.gov/pmc/articles/PMC3537499/
19. Vieira EM, Hasse M. Perceptions of professionals in an intersectorial network about the assistance of women in situation of violence. Interface. [Internet]. 2016;21(60):5162. [cited 2017 Jun 10]. Available from: http://www.scielo.br/ pdf/icse/v21n60/en_1807-5762-icse-1807-576220150357. pdf

20. Fukumitsu KO. O Psicoterapeuta diante do Comportamento Suicida. Psicologia USP. [Internet]. 2014; 25(3): 270-75. [cited 2017 Jun 10]. Available from: http://www.scielo.br/pdf/pusp/v25n3/0103-6564pusp-25-03-0270.pdf

21. Barbosa FO, Macedo PCM, Silveira RMC. Depressão e o suicídio. Rev. SBPH. [Internet]. 2011;14(1):233-43. [cited 2017 May 10]. Available from: http://pepsic.bvsalud. org/pdf/rsbph/v14n1/v14n1a13.pdf

22. Anestis MD, Soberay KA, Gutierrez PM, Hernández TD, Joiner TE. Reconsidering the Link Between Impulsivity and Suicidal Behavior. Pers Soc Psychol Rev. [Internet]. 2014;18(4):366-86. [cited 2017 May 10]. Available from: https://doi.org/10.1177/1088868314535988

23. Black DW, Coryell W, Crowe R, McCormick B, Shaw M, Allen J. Suicide Ideations, Suicide Attempts, and Completed Suicide in Persons with Pathological Gambling and their First-Degree Relatives. [Internet]. 2015; 45(6):700-709. [cited 2017 Abr 10]. Available from: https://www.ncbi.nlm. nih.gov/pmc/articles/PMC4595153/pdf/nihms664205.pdf 24. Goodterapy. Understanding Suicide: 5 Myths about Suicide. GoodTherapy.org Staff. [Internet]. 2014. [cited 2017 May 10]. Available from: https://www.goodtherapy. org/blog/understanding-suicide-5-myths-about-suicide/ 25. Hagen J, Hjelmeland H, Knizek BL. Relational Principles in the Care of Suicidal Inpatients: Experiences of Therapists and Mental Health Nurses. Issues Ment Health Nurs. [Internet]. 2017;38(2):99-106. [cited 2017 Abr 10]. Available from: doi: $10.1080 / 01612840.2016 .1246631$ 